\title{
Bortezomib-induced enzyme-targeted radiotherapy (BETR) for AIDS-related malignancies: efficacy assessment by Monte Carlo and dosimetry modeling
} R Hobbs*1, D Fu ${ }^{2}, \mathrm{M}$ Pomper $^{1}$, R Ambinder ${ }^{2}$ and G Sgouros ${ }^{1}$

\author{
Address: ${ }^{1}$ Department of Radiology, Johns Hopkins University, Baltimore, Maryland, USA and ${ }^{2}$ Department of Oncology, Johns Hopkins \\ University, Baltimore, Maryland, USA \\ * Corresponding author
}

\author{
from I I th International Conference on Malignancies in AIDS and Other Acquired Immunodeficiencies (ICMAOI): Basic, Epidemiologic, and Clinical \\ Research \\ Bethesda, MD, USA. 6-7 October 2008
}

Published: 17 June 2009

Infectious Agents and Cancer 2009, 4(Suppl 2):P23 doi:I0.II86/I750-9378-4-S2-P23

This abstract is available from: http://www.infectagentscancer.com/content/4/S2/P23

(C) 2009 Hobbs et al; licensee BioMed Central Ltd.

The Epstein-Barr virus (EBV) is associated with AIDSinduced lymphomas and Kaposi's sarcoma, and in most patients with these tumors, the viral genome serves as a nearly tumor-specific target. BETR therapy proposes to use Bortezomib to induce Epstein-Barr virus (EBV)-thymidine kinase (TK) expression, which can be targeted with iodine labeled 2'-fluoro-2'-deoxy-beta-D-5-iodouracil-arabinofuranoside (FIAU). Monte Carlo is an established procedure for simulating radioactive decay and obtaining the absorbed dose distribution from a spatial configuration of radionuclide decays. We present here the Monte Carlo simulation and dosimetry modeling of spherical tumors using GEANT4 for BETR to examine the issue of efficacy relative to percentage of cells induced to express viral TK, in particular for a level of 1 percent, which is the typical percentage of virally infected tumor cells for the AIDSinduced lymphomas.

We constructed a spherical tumor model of spherical cells ( $15 \mu \mathrm{m}$ diameter) in a hexagonal lattice of varying sizes ( $10,20,50$, and 150 cells per side). ${ }^{131}$ I is randomly allowed to decay according to three different models with varying degrees of ${ }^{131}$ I cell uptake: 100 percent of cells, 10 percent of cells, and 1 percent of cells containing ${ }^{131}{ }^{1}$. The energy deposited in each cell is collected and the dose to each cell calculated. Validation was performed, in part, by checking the MC-derived distribution of decaying ${ }^{131}$ I within the cells, and the spatial distribution of cells.
The model can be scaled to the tumor size by maintaining the correct ratio of decaying particles to cells and by increasing the amount of deposited energy per decay. The scaling factor comes from simple spherical modeling results, and reflects the fact that the ionizing particles have more distance to deposit energy as the tumor size increases. Also due to the large energy deposition range of ${ }^{131}$ I ( $\left.1 \mathrm{~mm}\right)$ compared to the cell size, we observe little difference in the dose distribution per cell between the different percentages of active cells.

The analysis suggests that potential therapeutic efficacy, as measured by the mean absorbed dose and spatial dose distribution in the tumor will be independent of the fraction of cells in the tumor that express viral TK and concentrate ${ }^{131}$ I-FIAU. The development of a kinetic model is the next step in this effort to assist in assessing the feasibility of porting Bortezomib-induced viral TK uptake of ${ }^{131} \mathrm{I}$ FIAU to clinical trials. The kinetic model features multistep kinetics including Bortezomib uptake, TK expression and [ ${ }^{131}$ I]FIAU absorption and retention. 